\title{
Giant Mediastinal Parathyroid Cyst with Hyperparathyroidism - a case report
}

\author{
Gabalec F. ${ }^{1}$, Svilias I. ${ }^{1}$, Hornychová H. ${ }^{2}$, Habal P. ${ }^{3}$, Čáp J. ${ }^{1}$ \\ ${ }^{1} 4$ th Dept. Of Internal Medicine, ${ }^{2}$ Fingerland's Department of Pathology ${ }^{3}$ Cardiosurgery clinic University Hospital in Hradec Kralove \\ and Charles University, Faculty of Medicine in Hradec Kralove, Czech Republic
}

\begin{abstract}
Primary hyperparathyroidism (PHPT) is rarely caused by parathyroid adenoma with cystic degeneration $(<1 \%)$, especially by the cyst localized in mediastinum.
\end{abstract}

Parathyroid gland cysts present $<0.01 \%$ of all neck masses

$10 \%$ of them localized in mediastinum

118 cases published all over the world until 2012
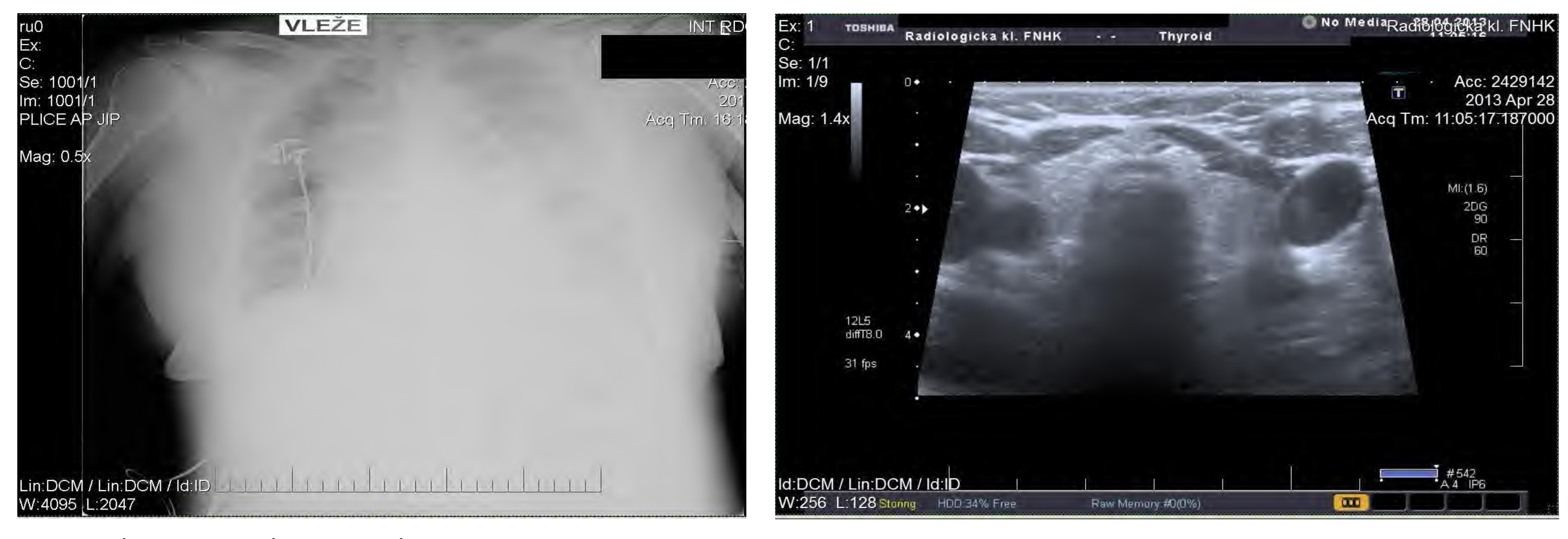

Fig. 1 Chest X-ray, horizontal position

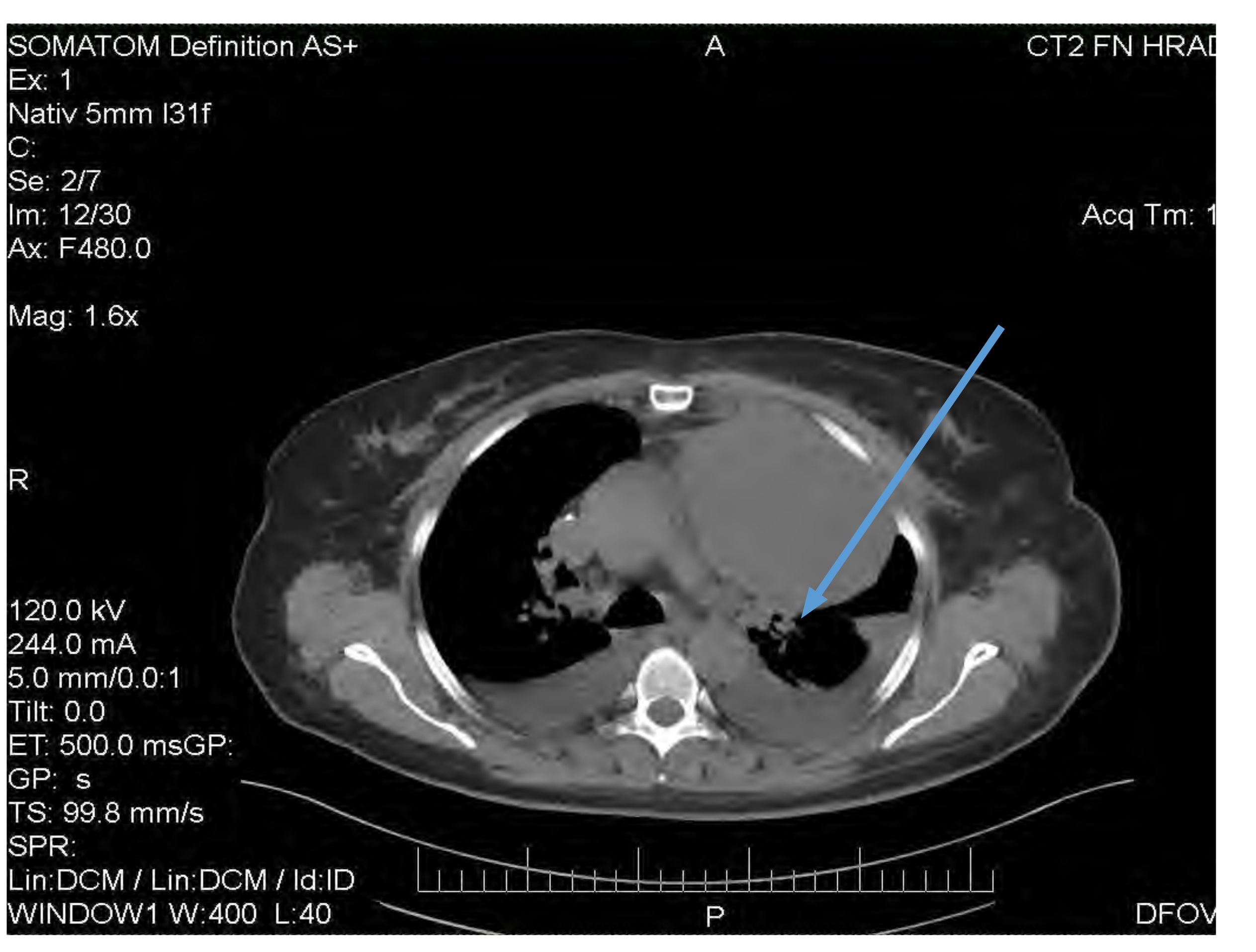

Fig. 3 Chest computer tomography with contrast transversal image

The situation suddenly became complicated with pancreatitis, symptoms from compression by large cyst and recurrent fever. Her calcium levels were only transiently stabilized after treatment with Cinacalcet (Mimpara) and Calcitonin.

A neck ultrasound (Fig.2), neck MRi and 99mTc_MIBI scintigraphy were with negative results. We performed again punction of the cyst, but the PTH level in the cyst fluid was lower $(4173 \mathrm{pg} / \mathrm{ml})$ than in serum $(6915 \mathrm{pg} / \mathrm{ml}$, normal range 13-50). When our patient became stable, without fever after antibiotic treatment, we performed left lateral thoracotomy with extirpation of the cyst. Parathyroid adenoma with cystic degeneration with residual atrophic thymic tissue was confirmed by histological examination.

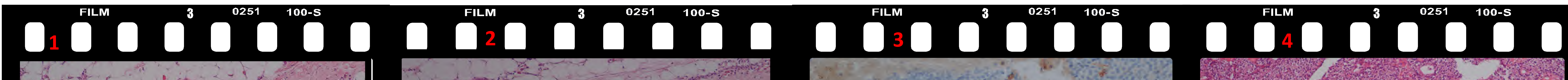

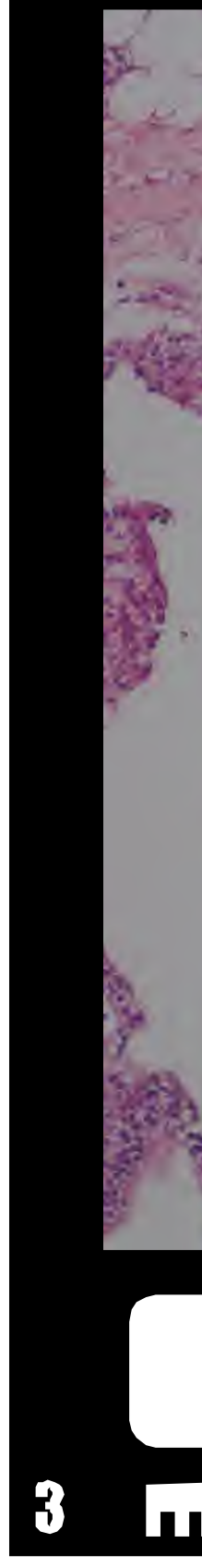 $1+2$ cyst of parathyroid gland and residual atrofic thymus tissue \\ 3 positivity of chromogranin - mosaic positivity is characteristic for parathyroid gland 4 Main parathyroid gland mass}

The serum calcium and also PTH are normalized after the surgery without any treatment and our patient is doing well. 